\title{
Parámetros de crecimiento de nuevos genotipos de arroz (Oryza sativa L.) en tres ambientes de Córdoba
}

\author{
Growth parameters of new genotypes of rice (Oryza sativa L.) \\ in three environments of Cordoba
}

\author{
Rogers E. Charry*, Dairo J. Pérez² ${ }^{2}$ Eliecer M. Cabrales³ ${ }^{3}$ Víctor Degiovanni ${ }^{4}$ \\ Recibido para publicación: Diciembre 5 de 2013 - Aceptado para publicación: Abril 18 de 2016
}

\begin{abstract}
RESUMEN
En Colombia, se han liberado genotipos de arroz adaptados a las variaciones del cambio climático, sin embargo, no se conoce su comportamiento en las principales zonas productoras de Córdoba. En este sentido, se evaluó la respuesta de los parámetros de crecimiento: Tasa relativa de crecimiento TRC, tasa de asimilación neta TAN, índice de área foliar IAF y relación de área foliar RAF, de los genotipos de arroz: Fedearroz Mocarí, Fedearroz Caracolí y Fedearroz 473, en varios ambientes del departamento de Córdoba (Lorica, Montería y San Bernardo del Viento). Se utilizó un diseño de bloques completos al azar en arreglo factorial $3 \times 3$, donde el primer factor fueron los genotipos y el segundo las tres localidades. Los resultados muestran influencia de la interacción variedad y localidad; donde Fedearroz Mocarí presentó el mejor comportamiento en TRC y TAN, Fedearroz Caracolí fue más estable en TRC y Fedearroz 473 en TAN. El comportamiento del IAF de Montería fue superior al de Lorica en 59,0 y 362,8\% al de San Bernardo. En Montería y San Bernardo Fedearroz Mocarí superó en 13,95 y 19,63\% a los demàs genotipos en RAF, mientras que en Lorica fue similar. Se concluye que Fedearroz Mocarí es la mejor opción a sembrar para los productores de arroz, por su eficiencia fotosintética en las tres zonas arroceras.
\end{abstract}

Palabras Clave: Cambio climático, genotipos, parámetros, eficiencia.

\begin{abstract}
In Colombia, new rice genotypes adapted to climate change have been released, however, their behavior is unknown in the main producing areas of Cordoba. In this sense, the response of the growth parameters was evaluated: relative growth rate (RGR), net assimilation rate (NAR), leaf area index (LAI) and leaf area ratio (LAR) of rice genotypes: Fedearroz Mocarí, Fedearroz Caracolí and Fedearroz 473, in various environments in the department of Córdoba (Lorica, Monteria and San Bernardo del Viento). Arandomized complete blocks design was used in a $3 \times 3$ factorial arrangement, in whichthe first factors were the genotypes and the second ones were the three locations. The results show the influence of the interaction between variety and location; where F. Mocarí madethe best performance in RGR and NAR, F. Caracolí was more stable in RGR and F. 473 in NAR. Monteria's LAI behavior was higher than Lorica's by $59.0 \%$ and San Bernardo's by $362.8 \%$. In Monteria and San Bernardo F. Mocarí topped by 13.95 and $19.63 \%$ the other genotypes in LAR, while in Lorica was similar. Therefore, it can be concluded that F. Mocarí is the best option for producers to plant rice, due to its photosynthetic efficiency evidenced in the three rice-growing areas.
\end{abstract}

Key Words: Climate change, genotypes, parameters, efficiency.

\footnotetext{
1* M.Sc. Fisiología Vegetal. Docente catedrático. Universidad de Córdoba, Montería-Colombia. E-mail: charry@fca.edu.co. Carrera 6 Nº 76-103, Monteria. Telefono: (+4) 7818010

${ }^{2}$ M.Sc. Fisiología Vegetal. Docente catedrático. Universidad de Córdoba, Montería-Colombia. E-mail: dairoperez81@hotmail.com

3 Ph.D. Ciencia del Suelo. Docente titular área de suelos. Universidad de Córdoba, Montería-Colombia. E-mail: ecabralesh@yahoo.es

${ }^{4}$ M.Sc. Fisiologia de Cultivos. Docente titular cultivo de arroz. Universidad de Córdoba, Montería-Colombia. E-mail: vmdegiovanni@ correo.unicordoba.edu.co
} 


\section{INTRODUCCIÓN}

El arroz (Oryza sativa L.) es el alimento básico de todos los paises de Asia y el Pacífico, África, América Latina y el Caribe, y una pequeña porción del Cercano Oriente. Si se consideran en conjunto todos los países en desarrollo, el arroz proporciona el $27 \%$ de la energía alimentaría y el $20 \%$ de la ingesta de proteína alimentaría (ONU 2002; ONU 2004: FAO 2006; IDEAS 2007; Degiovanni et al. 2010).

Colombia es responsable del $6,67 \%$ de la producción de arroz de America Latina. En el segundo semestre del 2015, la producción fue de 1.558.044 toneladas de arroz paddy verde, en un área cosechada de 295.971 hectáreas, de las cuales el 79,8\% correspondió a la siembra bajo el sistema de riego mecanizado y el $20,2 \%$ a secano mecanizado. A nivel nacional, el departamento del Casanare fue el de mayor producción, contribuyendo con el 35,13\%; seguido del Tolima con el $28,83 \%$, del resto de los departamentos con 19,29\%, entre los cuales se encuentra Córdoba, Meta y Huila con 15,04 y $6,71 \%$, respectivamente (FAO 2016; DANE 2016).

En los últimos años se han generado nuevos genotipos con capacidad de responder en el futuro al cambio climático ya que se prevé que los efectos de este, podrian ocasionar la desaparición de aquellos materiales geneticos susceptibles a esas nuevas condiciones en la región Caribe colombiana; esta estrategia ha tomado fuerza en los últimos años, considerándose la más viable frente a este fenomeno (Alexandrov 2000; Charlotte et al. 2013; Prior et al. 2011; Roudier 2011; Olesen et al. 2011); siendo importante evaluar esos genotipos bajo las condiciones climáticas de las diferentes regiones productoras de arroz, incluyendo las del departamento de Córdoba.

La capacidad de respuesta de los nuevos genotipos de arroz a las condiciones de variabilidad climática son evaluadas por medio de los índices de crecimiento, permitiendo conocer directamente el efecto del impacto ambiental en la fisiología de la planta, principalmente en la producción de biomasa y partición de sus asimilados (Beadle 1993). Los índices permiten describir el crecimiento cuantitativo, comparar la habilidad de un genotipo para crecer y desarrollarse en un ambiente dado en función del tiempo (Lambers et al. 2008; Flórez et al. 2010).

Los índices de crecimiento han servido para identificar la adaptación de genotipos en diferentes tipos de ambiente, siendo realizados un sinnúmero de investigaciones con diferentes genotipos de arroz; por ejemplo Maqueira et al. (2010) y Rebolledo et al. (2012), afirman que los genotipos de arroz que tienen altas tasas de tasa relativa de crecimiento (TRC), se caracterizan por un desarrollo rápido. Resultados de investigaciones de Osorio (2007) en genotipos de arroz en dos semestres, reportan una respuesta diferencial de la TRC de las variedades introducidas Blue Bonnet 50, IR 8 e IR 22; asimismo, que hubo un comportamiento similar de la tasa de asimilación neta (TAN) entre variedades, con tendencia a reducir progresivamente su valor desde etapas tempranas, a excepción del segundo semestre, cuando registraron un leve aumento de este parámetro.

Por otra parte, Osorio (2007) encontró que el Indice de area foliar (IAF) aumentó hasta la etapa de floración, a partir de donde comenzó a disminuir; sin embargo, Takai (2005) reportó similar tendencia, pero con un máximo incremento de este indice en el Ilenado del grano. Finalmente, Osorio (2007) encontró que el comportamiento de la razón de área foliar (RAF) fue diferencial entre semestres, reduciendo su valor desde la etapa vegetativa con un leve aumento en la etapa de floración en el primer semestre; $y$ un incremento significativo en el máximo macollamiento con una posterior 
disminución y un leve aumento en la etapa de embuchamiento para el segundo semestre.

En la selección de genotipos de arroz para una región determinada es importante evaluar su adaptabilidad en los dos semestres; entendiéndose como adaptabilidad la capacidad de un cultivo o planta de responder positivamente a los cambios de las condiciones ambientales como resultado de la interacción genotipo-ambiente, siendo controlada genéticamente, lo que permite aprovechar mejor la oferta ambiental (Cruz y Carneiro 2003; Chloupek y Hrstkova 2005).

La adaptabilidad de los cultivares de arroz a un ambiente, está determinada por su morfología y actividad metabólica, la cual puede variar según el genotipo y el estado de crecimiento del mismo (Shahidullah et al. 2009). Dos tipos de adaptabilidad son reconocidas, la general y la específica. En general, las variedades responden incrementando sus rendimiento bajo diversas condiciones climáticas y la específica es la capacidad de la planta de arroz de adaptarse a condiciones especiales favorables o adversas (Shahidullah et al. 2009).

El conocimiento de la adaptabilidad de los cultivares de arroz a determinados ambientes es de gran importancia para conocer su comportamiento agronómico, bien sea para fines de multiplicación de semilla o de producción de grano. Por lo tanto, el objetivo de la presente investigación fue evaluar la adaptabilidad de nuevos genotipos de arroz riego en las principales zonas productoras del departamento de Córdoba en respuesta al cambio climático.

\section{MATERIALES Y MÉTODOS}

Localización. El estudio se realizó durante el segundo semestre del 2012 en el departamento de Córdoba en los municipios de Montería $\left(8^{\circ}\right.$ $48^{\prime} \mathrm{N}$ y $\left.75^{\circ} 52^{\prime} \mathrm{W}\right)$, Lorica $\left(9^{\circ} 4^{\prime} \mathrm{N}\right.$ y $75^{\circ} 50 \mathrm{~W}$ ) y San Bernardo del Viento $\left(9^{\circ} 13^{\prime} \mathrm{N}\right.$ y $\left.75^{\circ} 54^{\prime} \mathrm{W}\right)$ (Palencia et al. 2006). Por razones fitosanitarias, se estableció el experimento durante este periodo, que luego fue reglamentado por la resolución ICA N²23 del 19 agosto 2014.

Material vegetal. Los genotipos estudiados correspondieron a las variedades de arroz Fedearroz Mocarí, Fedearroz Caracolí y Fedearroz 473, siendo esta última, el testigo por su estabilidad y rendimiento en el departamento de Córdoba.

Diseño experimental. Se utilizó un diseño de bloques completos al azar en arreglo factorial $3 \times 4$ con tres repeticiones, donde el primer factor correspondió a las variedades: Fedearroz Mocarí, Fedearroz Caracolí y Fedearroz 473, y el segundo factor, a las localidades: Montería, Lorica y San Bernardo del Viento. Cada unidad experimental contó con un área de $30 \mathrm{~m} 2$, donde las plantas fueron sembradas por trasplante con un arreglo espacial de $30 \mathrm{~cm}$ entre plantas y surcos, para una densidad de 111.111 plantas/ha.

Se evaluaron los parámetros de crecimiento: tasa relativa de crecimiento (TRC), tasa de asimilación neta (TAN), índice de área foliar (IAF) y razón de área foliar (RAF). Para ello, se realizaron muestreos destructivos cada 8 días, tomando al azar 3 plantas por parcela para la determinación de masa seca y área foliar. En el material extraído, el área foliar se estimó a través de la relación peso: área, registrando su peso en fresco y el peso de 10 círculos cortados con un sacabocado de área conocida $(0,28 \mathrm{~cm} 2)$ y la materia seca mediante la deshidratación de la muestra en una estufa durante 72 horas a $70{ }^{\circ} \mathrm{C}$. Con esta información se calcularon los parámetros de crecimiento, de acuerdo a las fórmulas utilizadas por Jarma et al. (2010).

Análisis de datos. Los datos obtenidos se analizaron mediante un análisis de varianza (ANAVA), previa verificación y cumplimiento de 
los supuestos de normalidad y homogeneidad de varianzas con las pruebas de Shapiro-Wilk $(p \leq 0,05)$ y Levene $(p \leq 0,05)$, respectivamente. La interacción de los factores fue analizada con la curva de cada genotipo en función de la localidad (ambiente), seleccionando el modelo de regresión de mejor ajuste, tomando como referencia el mayor coeficiente de determinación $\left(\mathrm{R}^{2}\right)$. El análisis de datos se realizó con el programa estadístico SAS (Statistical Analisys System), versión 9.1 (SAS, 1999).

\section{RESULTADOS Y DISCUSIÓN}

El ANAVA indica que hubo efectos significativos $(p \leq 0.05)$ y altamente significativos $(p \leq 0.01)$ en la interacción entre los factores variedad y localidad en todos los parámetros de crecimiento. Asimismo, estos factores por separado mostraron tendencia a reportar efectos significativos (Tabla 1).

Tasa relativa de crecimiento (TRC). La respuesta de la TRC de los genotipos fue diferencial entre las localidades de Lorica, Monteria y San Bernando del Viento (Figura 1a, 1b y 1c). Asimismo, esta fue explicada por modelos de regresión de segundo orden (Tabla 2).

En la localidad de Lorica, las variedades Fedearroz Mocarí y Fedearroz 473 tuvieron un comportamiento similar y diferente a Fedearroz Caracolí. La TRC de los genotipos F. Mocarí y F. 473 se incrementó hasta el inicio del primordio

Tabla 1. Cuadrado medio y nivel de significancia del análisis de varianza de evaluaciones de los parámetros de crecimiento TRC, TAN, RAF y IAF de tres variedades de arroz en igual número de ambientes.

\begin{tabular}{|c|c|c|c|c|c|c|c|c|c|c|c|c|}
\hline \multirow{2}{*}{ Parámetro } & \multirow[b]{2}{*}{ Fuente de Variación } & \multirow[b]{2}{*}{ gl } & \multicolumn{10}{|c|}{ Días después de la emergencia } \\
\hline & & & 41 & \multicolumn{2}{|l|}{48} & \multicolumn{2}{|l|}{55} & \multicolumn{2}{|l|}{69} & 76 & \multicolumn{2}{|c|}{83} \\
\hline & Bloque & 2 & $0,0012 \mathrm{~ns}$ & \multicolumn{2}{|l|}{$0,0097^{* *}$} & \multicolumn{2}{|c|}{$0,000095^{* *}$} & \multicolumn{2}{|c|}{$0,000012 \mathrm{~ns}$} & $0,000094^{*}$ & \multicolumn{2}{|c|}{$0,0029^{*}$} \\
\hline & Variedad (V) & 2 & $0,0056^{* *}$ & \multicolumn{2}{|l|}{$0,0070^{*}$} & \multicolumn{2}{|c|}{$0,000001 \mathrm{~ns}$} & \multicolumn{2}{|c|}{$0,000199^{* *}$} & $0,000078^{* *}$ & \multicolumn{2}{|c|}{$0,0020^{*}$} \\
\hline \multirow[t]{5}{*}{ TRC } & Localidad (L) & 2 & $0,0048^{* *}$ & \multicolumn{2}{|c|}{$0,00608^{*}$} & \multicolumn{2}{|c|}{$0,000099^{*}$} & \multicolumn{2}{|c|}{$0,000110^{*}$} & $0,000036 \mathrm{~ns}$ & \multicolumn{2}{|c|}{$0,0050^{* *}$} \\
\hline & Interacción $\mathrm{V}^{*} \mathrm{~L}$ & 4 & $0,0060^{* *}$ & \multicolumn{2}{|c|}{$0,0093^{* *}$} & \multicolumn{2}{|c|}{$0,000087^{*}$} & \multicolumn{2}{|c|}{$0,000173^{* *}$} & $0,000050^{*}$ & \multicolumn{2}{|c|}{$0,0043^{* *}$} \\
\hline & Error & 14 & 0,0008 & \multicolumn{2}{|l|}{0,0018} & \multicolumn{2}{|c|}{0,000023} & \multicolumn{2}{|c|}{0,000028} & 0,000015 & \multicolumn{2}{|c|}{0,00049} \\
\hline & Bloque & 2 & $2,7 \times 10-7 \mathrm{~ns}$ & \multicolumn{2}{|c|}{$9,0 \times 10-8 \mathrm{~ns}$} & \multicolumn{2}{|c|}{$3,9 \times 10-8^{*}$} & $1,1 \times 10-7$ & & $2,2 \times 10-8 *$ & & $x 10-8^{*}$ \\
\hline & Variedad (V) & 2 & $6,5 \times 10-7^{* *}$ & $1,5 \times 10-7$ & & $2,5 \times 10-$ & $8 \mathrm{~ns}$ & $2,1 \times 10-7$ & & $2,0 \times 10-8^{*}$ & $3,3 x$ & x10-8ns \\
\hline TAN & Localidad (L) & 2 & $4,0 \times 10-7 *$ & $3,1 \times 10-7$ & & $3,4 \times 10-$ & & $1,9 \times 10-7$ & & $3,9 \times 10-8^{* *}$ & $3,8 x$ & $x 10-8^{*}$ \\
\hline & Interacción $\mathrm{V}^{*} \mathrm{~L}$ & 4 & $4,3 \times 10-7^{*}$ & $2,7 \times 10-7$ & & $6,5 \times 10-$ & $8^{* *}$ & $0,9 \times 10-7$ & & $4,1 * \times 10-8^{*}$ & $6,9 x$ & $x 10-8^{* *}$ \\
\hline & Error & 14 & $9,1 \times 10-8$ & $3,0 \times 10-8$ & & $8,1 \times 10-$ & & $2,0 \times 10-8$ & & $5,0 \times 10-9$ & $9,0 x$ & $x 10-9$ \\
\hline & Bloque & 2 & $42 \mathrm{~ns}$ & $16,2 \mathrm{~ns}$ & & $91,1^{* *}$ & & $121,4 \mathrm{~ns}$ & & $109,4^{*}$ & $72,8^{*}$ & \\
\hline & Variedad (V) & 2 & $64^{*}$ & $65,9^{* *}$ & & $78,4^{*}$ & & $308,7^{* *}$ & & $236,9^{* *}$ & $84,3^{3}$ & \\
\hline RAF & Localidad (L) & 2 & $123^{* *}$ & $36,1^{*}$ & & $83,5^{* *}$ & & $169,3^{*}$ & & $98,4^{*}$ & $104,6^{\prime}$ & \\
\hline & Interacción $\mathrm{V}^{*} \mathrm{~L}$ & 4 & $147^{* *}$ & $37,8^{*}$ & & $90,9^{* *}$ & & $323,2^{* *}$ & & $101.5^{*}$ & $109,2^{*}$ & \\
\hline & Error & 14 & 15,3 & 8,7 & & 19,6 & & 43,9 & & 24,6 & 9,3 & \\
\hline & & & & & & Días de & spués de la & a emergenc & & & & \\
\hline Parámetro & Fuente de Variación & gl & 14 & 28 & & & 49 & 56 & 70 & 84 & 98 & 114 \\
\hline IAF & Bloque & 2 & $2,4 \times 10-7 \mathrm{~ns}$ & $9,7 \times 10-5 \mathrm{~ns}$ & 1,3 & $<10-4 *$ & $0,0223^{* *}$ & $0,78^{* *}$ & $0,033^{*}$ & $0,63^{* *}$ & $1,76^{* *}$ & $3,92^{*}$ \\
\hline & Variedad (V) & 2 & $2,3 \times 10-7 \mathrm{~ns}$ & $1,95 \times 10-5 \mathrm{~ns}$ & 7,6 & $0-5 \mathrm{~ns}$ & $0,0189^{* *}$ & $0,83^{* *}$ & $0,036^{*}$ & $0,45^{* *}$ & $1,45^{* *}$ & $3,63^{*}$ \\
\hline & Localidad (L) & 2 & $2,0 \times 10-7 \mathrm{~ns}$ & $1,81 \times 10-5 \mathrm{~ns}$ & 1,2 & $\times 10-4 n s$ & $0,0102 \mathrm{~ns}$ & $0,72 \mathrm{~ns}$ & $0,029 \mathrm{~ns}$ & is $0,56 \mathrm{~ns}$ & $1,89^{* *}$ & $3,52^{*}$ \\
\hline & Interacción $\mathrm{V}^{*} \mathrm{~L}$ & 4 & $1,2 \times 10-8 \mathrm{~ns}$ & $2,9 \times 10-5 \mathrm{~ns}$ & 4,3 & $0-5 \mathrm{~ns}$ & $0,0001 \mathrm{~ns}$ & $0,05 \mathrm{~ns}$ & $0,014 \mathrm{~ns}$ & s $2,5^{*}$ & $0,96^{*}$ & $5,98^{* *}$ \\
\hline & Error & 1 & $1,1 \times 10-8$ & $2,3 \times 10-5$ & 1,6 & 10-5 & 0,0025 & 0,08 & 0,007 & 0,54 & 0,02 & 0,87 \\
\hline
\end{tabular}

gl: grados de libertad; $* * *$ : diferencias significativas $(p \leq 0,05)$ y altamente significativas $(p \leq 0,01)$, respectivamente; ns: diferencias no significativas. Tasa relativa de crecimiento TRC. Tasa de asimilación neta TAN. Razón de área foliar RAF. Índice de área foliar IAF. 
Tabla 2. Modelos de regresión de los parámetro de crecimiento de tres variedades de arroz en igual número de localidades del departamento de Córdoba.

\begin{tabular}{|c|c|c|c|c|}
\hline Parámetro & Localidad & Variedad & Modelo de regresión & $\mathbf{R}^{2}$ \\
\hline & Lorica & F. Caracolí & $Y=0,12-0,003 X-0,000015 X 2$ & 0,78 \\
\hline & & F. Mocarí & $Y=-0,40+0,02 X-0,0001 X 2$ & 0,76 \\
\hline & & F. 473 & $Y=-0,60+0,023 X-0,0001 X 2$ & 0,74 \\
\hline \multirow[t]{9}{*}{ TRC } & Montería & F. Caracolí & $Y=-0,49+0,02 X+0,0001 X 2$ & 0,81 \\
\hline & & F. Mocarí & $Y=-1,47-0,04 X+0,00002 X 2$ & 0,84 \\
\hline & & F. 473 & $Y=-0,38+0,02 X-0,0001 X 2$ & 0,92 \\
\hline & San Bernardo & F. Caracolí & $Y=-1,13+0,04 X-0,0003 X 2$ & 0,82 \\
\hline & & F. Mocarí & $Y=-0,78+0,03 X-0,0003 X 2$ & 0,77 \\
\hline & & F. 473 & $Y=-1,33+0,05 X-0,0004 X 2$ & 0,60 \\
\hline & Lorica & F. Caracolí & $Y=0,0013-0,0001 X$ & 0,99 \\
\hline & & F. Mocarí & $Y=-0,01-0,0003 X+2,2 * 10 E-6 X 2$ & 0,87 \\
\hline & & F. 473 & $Y=-0,01+0,0004 X-3,4 * 10-6 \times 2$ & 0,78 \\
\hline \multirow[t]{9}{*}{ TAN } & Montería & F. Caracolí & $Y=0,0006-2,9 * 10-5 X-4,3 * 10-7 X 2$ & 0,84 \\
\hline & & F. Mocarí & $Y=0,002-1,4^{*} 10-5 X-1,7^{*} 10-7 X 2$ & 0,78 \\
\hline & & F. 473 & $Y=-0,0006+5,9^{*} 10-5 X-6,0 * 10-7 X 2$ & 0,87 \\
\hline & San Bernardo & F. Caracolí & $Y=-0,007+0,0003 X-2,5 * 10-6 X 2$ & 0,71 \\
\hline & & F. Mocarí & $Y=-0,0005+6,6 * 10-5 X-7,5 * 10-7 X 2$ & 0,56 \\
\hline & & F. 473 & $Y=-0,009+0,0003 X-2,7^{*} 10-6 X 2$ & 0,79 \\
\hline & Lorica & F. Caracolí & $Y=0,4774-0,0527 X+0,0012 X 2-5,8388 * 10-6 X 3$ & 0,87 \\
\hline & & F. Mocarí & $Y=0,2213-0,0247 X+0,0006 X 2-2,5027^{*} 10-6 X 3$ & 0,94 \\
\hline & & F. 473 & $Y=0,6544-0,0730 X+0,0017 X 2-9,0824 * 10-6 X 3$ & 0,78 \\
\hline \multirow[t]{9}{*}{ IAF } & Montería & F. Caracolí & $Y=2,2456-0,2666 X+0,0067 X 2-3,549 * 10-5 X 3$ & 0,6 \\
\hline & & F. Mocarí & $Y=1,5389-0,2042 X+0,0057 X 2-3,1923 * 10-5 X 3$ & 0,83 \\
\hline & & F. 473 & $Y=2,1077-0,2517 X+0,0065 X 2-3,6145 * 10-5 X 3$ & 0,91 \\
\hline & San Bernardo & F. Caracolí & $Y=1,5833-0,2152 X+0,0062 X 2-3,8956 * 10-5 X 3$ & 0,48 \\
\hline & & F. Mocarí & $Y=1,3693-0,2039 X+0,0063 X 2-4.1466 * 10-5 X 3$ & 0,59 \\
\hline & & F. 473 & $Y=1,5576-0,2075 X+0,0059 \times 2-3,7050 * 10-5 X 3$ & 0,6 \\
\hline & Lorica & F. Caracolí & $Y=159,32-6,5711 X$ & 0,99 \\
\hline & & F. Mocarí & $Y=294,59-3,99 X+0,021 X 2$ & 0,65 \\
\hline & & F. 473 & $Y=290,20-3,58 X+0,017 X 2$ & 0,68 \\
\hline \multirow[t]{6}{*}{ RAF } & Montería & F. Caracolí & $Y=-353,86+16,05 X-0,11 X 2$ & 0,76 \\
\hline & & F. Mocarí & $Y=-671,39+28,14 X-0,22 X 2$ & 0,65 \\
\hline & & F. 473 & $Y=-389,60+17,61 X-0,13 X 2$ & 0,52 \\
\hline & San Bernardo & F. Caracolí & $Y=-2036,06+71,05 X-0,52 X 2$ & 0,71 \\
\hline & & F. Mocarí & $Y=-2297,28+83,08 X-0,62 X 2$ & 0,69 \\
\hline & & F. 473 & $Y=-1331,67+48,39 X-0,36 X 2$ & 0,65 \\
\hline
\end{tabular}

floral (62 días después de la emergencia dde), a partir de allí, este parámetro tendió a disminuir hasta la floración. Por otra parte, en la fase vegetativa F. Caracolí registró la mayor TRC inicial (41 dde), la cual se redujó significativamente hasta la floración (Figura 1a).

En Montería, la variedad F. Mocarí tuvo un comportamiento diferencial al resto de los materiales. F. Caracolí y F. 473 incrementaron su TRC hasta la etapa de inicio del primordio floral (55 dde), luego esta dismunuyó hasta la floración. En cambio, F. Mocarí presentó una TRC superior al resto de los genotipos a los 41 dde (fase vegetativa), que posteriormente tendió a reducirse, donde fue más significativa hasta el inicio del primordio floral entre 41 y 62 dde (Figura 1b). 
En San Bernardo del Viento, los genotipos mostraron similar tendencia, en la cual la TRC se incrementó hasta la la etapa de inicio del primordio floral, punto a partir del cual fue disminuyendo hasta registrar valores muy bajos. En F. Caracolí y F. Mocarí la máxima TRC ocurrió a los 55 dde y en F. 473 a los 62 dde (Figura 1c).

Estos resultados coinciden parcialmente con los encontrados por Osorio (2007), para la TRC de las variedades introducidas Blue Bonnet 50, IR 8 e IR 22, donde la TRC de IR 22 se incrementó significativamente alrededor de los 45 dde y posteriormente disminuyó durante la etapa de floración. Según Maqueira et al. (2010), Nargis y Golam (2011) y Rebolledo et al. (2012), esta respuesta obedece al incremento de la biomasa asociada al número de macollas y el incremento del área foliar, a finales de la fase vegetativa y desarrollo de la fase reproductiva. Rebolledo et al. (2012) afirma que aquellos genotipos que tienen valores altos de TRC se caracterizan por un desarrollo rapido, tal como ocurrió con algunas variedades en las tres localidades evaluadas.

Tasa de Asimilación Neta (TAN). La TAN de las variedades tendió a mostrar una respuesta diferencial entre localidades, que se explicó por modelos de regresión de segundo orden, a excepción del genotipo F. Caracolí en condiciones de Lorica, quien tuvo un comportamiento de orden lineal (Tabla 2).

En Lorica, la TAN de F. 473 se incrementó significativamente hasta el inicio del primordio foliar (62 dde) y a partir de este punto, disminuyó cuadraticamente. En F. Mocarí la TAN se redujo tempranamente (desde la fase vegetativa) hasta incrementarse levemente en la etapa de floración. Por otra parte, la TAN de F. Caracolí disminuyó linealmente desde la etapa vegetativa hasta la etapa de floración (Tabla 2 y Figura 1d).

En Montería, los genotipos F. Mocarí y F. Caracolí tuvieron similar respuesta, donde la TAN dismuyó desde la etapa vegetativa conforme el desarrollo del cultivo, siendo el primero y el segundo los de mayor valor inicial y reducción de capacidad fotosintetica, respectivamente. El genotipo F. 473 a diferencia del resto del recurso genetico, tuvo un leve incremento en la etapa vegetativa, es decir, hasta los 48 dde (Figura 1e).

En San Bernardo del Viento, la TAN de F. Caracolí y F. 473 se incrementó hasta estados avanzados de la etapa de desarrollo del primordio floral (55 dde) y etapa de embuchamiento (62 dde), respectivamente; a partir de donde disminuyó drasticamente. En contraste, la TAN de F. Mocarí disminuyó de forma temprana en la fase vegetativa, es decir a los 41 dde (Figura 1f).

Estos resultados coinciden parcialmente con Osorio (2007), quien después de evaluar en dos semestres varios genotipos, reporta un comportamiento similar entre variedades, con tendencia a reducir su TAN progresivamente desde etapas tempranas hasta el final del cultivo, a excepción del semestre B, cuando hubo un leve aumento de este parámetro en la floración. Según Mora et al. (2006), la disminución de la TAN después de alcanzar el máximo valor, indica que la capacidad fotosintética del dosel tiende a disminuir continuamente con el crecimiento y expansión del área foliar y puede cesar en el momento en que las plantas alcanzan su máxima área foliar, debido principalmente, al sombreo que ocurre entre las hojas de los diferentes estratos del dosel, lo cual ocasiona que la respiración tienda a ser mayor que la fotosíntesis.

Índice de área foliar (IAF). El IAF de las variedades de arroz en los diversos ambientes, se explicó por un modelo de regresion de orden cubico (Tabla 2). En las figuras $1 \mathrm{j}, 1 \mathrm{k}$ y $1 \mathrm{I}$, se puede apreciar que el IAF en la fase vegetativa disminuyó por el estrés ocasionado por la aclimatación (trasplante), pero luego, este se incrementó significativamente hasta la fase reproductiva y de maduración; a partir de 
donde se redujo con la formación del grano.

En Lorica, el IAF de F. Mocarí y F. Caracolí se incrementó a partir de la fase vegetativa (entre 42 y 49 dde), donde se observaron leves incrementos del área foliar, aún en las etapas finales de la fase de maduración del grano. El IAF de F. 473 aumentó significativamente entre la etapa vegetativa y la etapa lechosa del grano (entre 49 y 98 dde), a partir de donde mostró un patrón de reducción (Figura 1j).

En Monteria, el IAF de todos los genotipos se incrementó a partir de la etapa de máximo macollamiento (entre 35 y 42 dde) hasta alcanzar su mayor valor, el cual para F. Mocarí y F. 473 ocurrió en la etapa lechosa del grano (98 dde) y para F. Caracolí en la etapa pastosa (105 dde). Después de este punto, este parámetro se redujo (Figura $1 \mathrm{k}$ ). Por otra parte, la respuesta de San Bernado del Viento fue similar a la descrita para Montería, pero a diferencia de que el máximo IAF se alcanzó en la etapa de desarrollo de la panicula (Figura 1l).

El IAF de las localidades de Monteria y San Bernardo del Viento fue superior al registrado en Lorica. Los tres materiales estudiados, sembrados en Montería y San Bernardo del Viento, presentaron un IAF con tendencia de decrecer un poco después de la floración (Figura $1 \mathrm{j}, 1 \mathrm{k}$ y $1 \mathrm{l}$ ). Los valores de IAF para Montería y San Bernardo del Viento coinciden con los rangos considerados como óptimos para el cultivo, siendo entre 4-8 los óptimos (Riveros 1994; Osorio 2007; Maqueira et al. 2007; Maqueira et al. 2009; Maqueira et al. 2010). Por otra parte, Hernández y Soto (2012), Soto y Hernández (2012), indican que bajos rangos de IAF (menores de 2) limitan la intercepción de la radiación solar y por consiguiente la producción de biomasa; situación que se reflejó en Lorica.

La tendencia del IAF de los genotipos evaluados coincide con Osorio (2007), donde los genotipos aumentaron su IAF hasta floración, etapa a partir de donde comenzó a disminuir; pero difieren con Takai (2005), quien reportó que el máximo incremento del IAF ocurrio con el Ilenado del grano. Según Riveros (1994), Maqueira et al. (2010) el IAF se reduce después de alcanzar su máximo valor por la muerte de las hojas inferiores por senescencia.

Relación de área foliar (RAF). La respuesta de la RAF de los genotipos de arroz tuvo un comportamiento de segundo orden, que fue similar en las condiciones de Montería y San Bernardo del Viento, y a su vez, diferente al observado en Lorica, donde la respuesta fue explicada por modelos de primer y segundo orden (Tabla 2).

En Lorica, la RAF de F. Caracolí fue explicada por un modelo de regresión lineal y la de F. Caracolí y F. 473 por un modelo de segundo orden. Independiente del modelo, la RAF mostró un patrón de disminución a partir de la fase vegetativa (Tabla 2 y Figura 1g).

En Monteria, a partir de la fase vegetativa la RAF se incrementó linealmente, pero después de alcanzar su máximo valor se redujo (Tabla 2). En este sentido, los genotipos F. Caracolí y F. 473 aumentaron su RAF hasta la etapa de inicio del primordio floral (62-69 dde) y F. Mocarí hasta el desarrollo de la panicula, es decir a los 76 dde (Figura 1h).

En San Bernardo delViento, la RAF se incrementó hasta el desarrollo de la panicula (69 dde) para todos los materiales, siendo F. Mocarí el genotipo que alcanzó el mayor valor (Figura $1 \mathrm{i}$ ). Para la localidad de Lorica, el comportamiento de la variable RAF decrece con la edad del cultivo. La respuesta de la RAF de los nuevos genotipos de arroz coincide parcialmente con lo afirmado por Osorio (2007), quien reporta que esta variable se incrementó significativamente hasta el máximo macollamiento, desde donde disminuyó drasticamente y registró un leve aumento en la etapa de embuchamiento. 

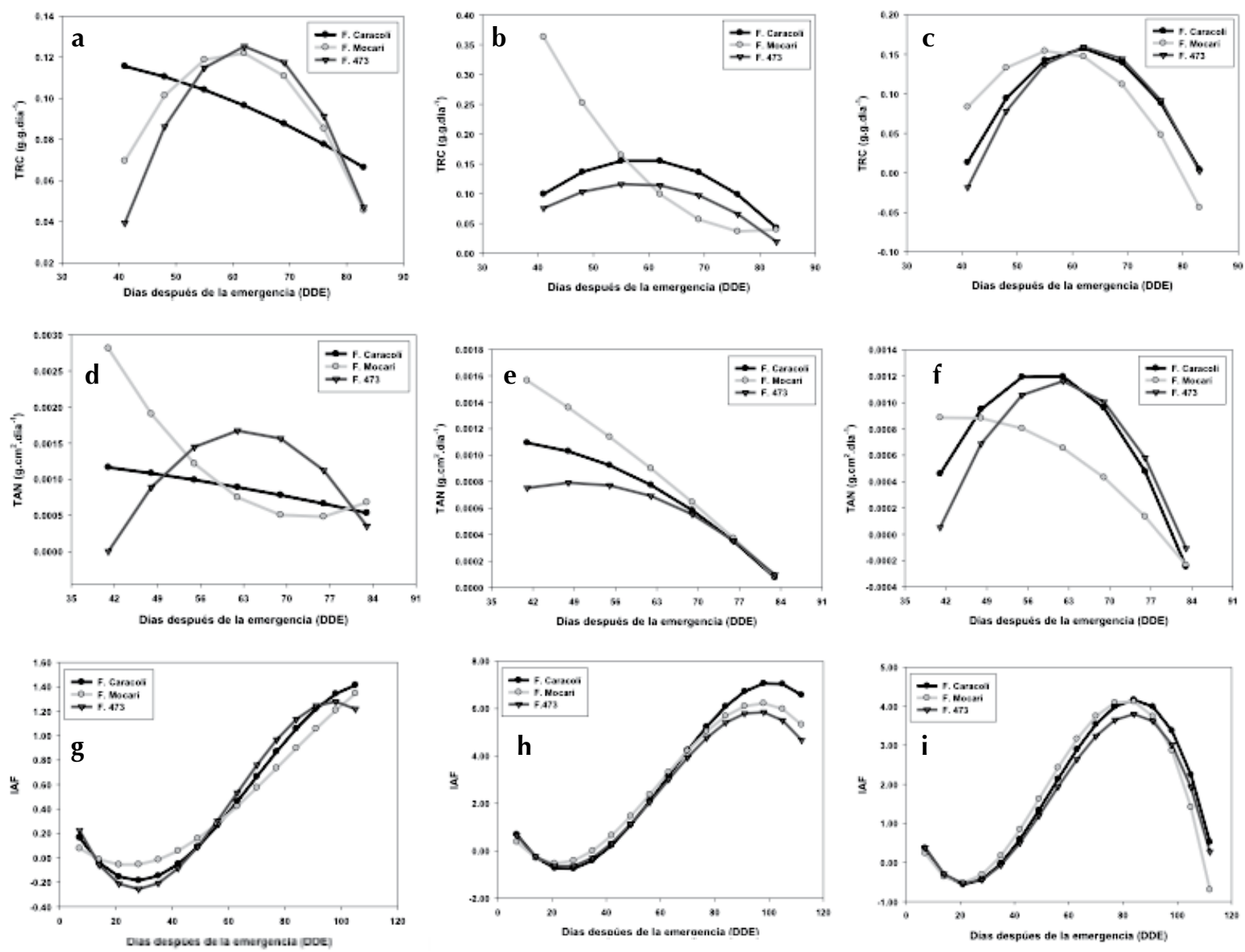

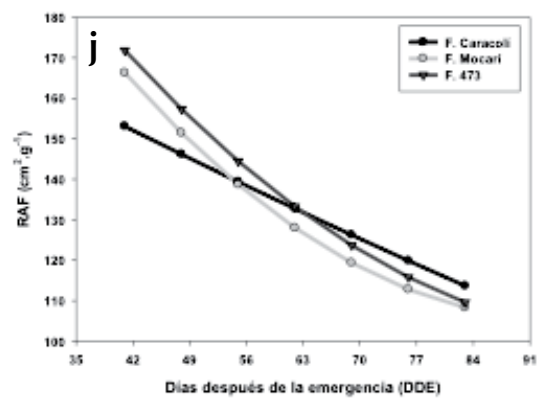

Lorica

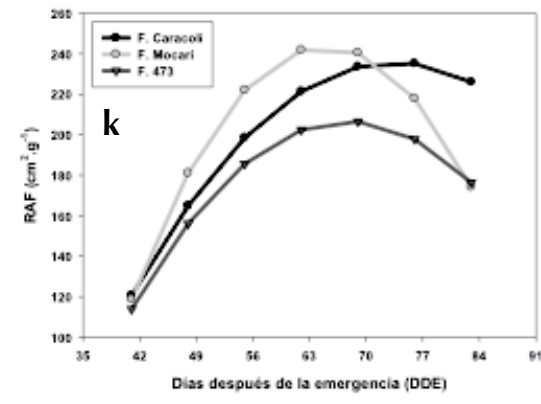

Montería



San Bernardo del Viento

Figura 1: Parámetros de crecimiento de tres variedades de arroz en tres localidades de Córdoba. Lorica: a) TRC, d) TAN, j) IAF, g) RAF; Montería: b) TRC, e) TAN, k) IAF, h) RAF; San Bernardo del Viento: c) TRC, f) TAN, I) IAF, i) RAF.

\section{CONCLUSIONES}

La variedad Fedearroz Mocarí registró el mejor comportamiento de los parámetros de eficiencia fotosintetica (TRC y TAN) en todas las localidades, siendo la de mejor estabilidad en cuanto a la respuesta en las variaciones de los factores ambientales. Lo que sugiere que los rendimientos esperados serán superiores, posicionando a este material como la mejor alternativa de siembra comercial para los productores de la región. 


\section{REFERENCIAS}

Alexandrov, V. and Hoogenboom, Y. 2000. The impact of climate variability and change on crop yield in Bulgaria. Agricultural and Forest Meteorology 104(4): 315-32.

Beadle, C. 1993. Growth analysis. In: Hall, D., Scurlock, J., Bolhar, H., Nordem, K., Leegood, R. y Long, S. (Ed). Photosynthesis an production in a changing enviroment: A field and laboratory manual. Chapman \& Hall, London, p 36-46.

Charlotte, L., Jarvis, A. y Ramirez, J. 2013. Agricultura colombiana: Adaptación al cambio climático. https://ciat.cgiar.org/ es/publication/agricultura-colombianaadaptacion-al-cambio-climatico [17 de enero 2016].

Chloupek, O., and Hrstkova, P. 2005. Adaptation of crops to environment. Theoretical and Applied Genetic 111 (7): 1316-1321.

Cruz, C. y Carneiro, P. 2003. Modelos biométricos aplicados ao melhoramento genético (volume 2). Editora UFV, Viçosa, 585 p.

Departamente Administrativo Nacional de Estadística - DANE. 2016. Boletín Técnico: Encuesta nacional de arroz mecanizado: II Semestre de 2015. http://www.dane.gov.co/index.php/ agropecuario-alias/estadisticas-de-arrozmecanizado-enam [17 de mayo 2016].

Degiovanni V., Berrio, L. y Charry, R. 2010. Origen, taxonomía, anatomía y morfología de la planta de arroz (Oryza sativa L.). En: Degiovanny, V., Martínez, C. y Motta, F. Producción Eco-eficiente del Arroz en América Latina. Tomo I. CIAT, Cali, p 35-59
FAO. 2006. Perspectivas alimentarias: Análisis del mercado mundial. http://www.fao. org/docrep/009/j7927s/j7927s17.htm [12 de febrero 2016].

FAO. 2016. Rice production statistics 2013. http://www.fao.stat.org [1 de enero 2016].

Hernández, C. y Soto, C. 2012. Influencia de la fecha de siembra sobre el crecimiento y la relación fuente-demanda del cultivo del Maíz (Zea mays L.). Cultivos Tropicales 33 (1): 28-34.

Iniciativa de economía alternativa y solidaria - IDEAS. 2007. La producción y el comercio internacional del arroz Boletín N¹6. IDEAS, España, p 1-56.

Jarma, A., Degiovanny, V. y Montoya R. 2010. Índices fisiotécnicos, fases de crecimiento y etapas de desarrollo de la planta de arroz. En: Degiovanny, V., Martínez, C. y Motta, F. Producción Eco-eficiente del Arroz en América Latina. Tomo I. CIAT, Cali, p 60-82.

Lambers, H., Chapin, S. and Pons, T. 2008. Plant physiological ecology. 2nd ed. Springer-Science, New York, 223 p.

Maqueira, L., Torres, W., Díaz, G. y Torres, K. 2007. Efectos del sistema intensivo del cultivo arrocero (SICA) sobre algunas variables de crecimiento y el rendimiento en una variedad de ciclo corto. Cultivos Tropicales 28 (2): 59-61.

Maqueira, L., Torres, W. y Miranda, A. 2009. Crecimiento y rendimiento de dos variedades de arroz de ciclo corto en época poco Iluviosa. Cultivos Tropicales 30 (3): 28-31.

Maqueira, L., Pérez, S. y Torres, W. 2010. Crecimiento y Productividad de variedades de arroz de diferentes ciclos 
en dos fechas de Siembra en la época de frío en los Palacios, Pinar del Río. Cultivos Tropicales 31 (4): 2-8.

Mora, R., Ortiz, J., Rivera A., Mendoza, M., Colinas, T. y Lozoya, H. 2006. Índices de eficiencia de genotipos de papa establecidos en Condiciones de secano. Revista Chapingo 12 (1): 85-94.

Nargis, J. and Golam, A. 2011. Comparative growth analysis of two varieties of rice following Naphthalene Acetic Acid Application. Journal of Bangladesh Academy of Sciences 35 (1): 113-120.

Olesen, J., Trnka, M., Kersebaum, K., Skjelvåg, A., Seguin, P., Peltonen-Sainio, P., Rossi, F., Kozyra, J. and Micale, F. 2011. Impacts and adaptation of European crop production systems to climate change. Europ. J. Agronomy 34: 96-112.

Organización de las Naciones Unidas ONU. 2002. Noticiario de la comisión internacional del arroz Vol. 51. FAO, Rome. p 1-89.

Organización de las Naciones Unidas - ONU. 2004. $20^{a}$ Conferencia regional de la FAO para America Latina y el Caribe: Año internacional del arroz. FAO, Guatemala, p 1-26.

Osorio, J. 2007. Evolución del crecimiento, rendimiento de grano y partición de fotosintatos en 14 variedades de arroz representantes de diversos ciclos de mejoramiento en Colombia. Tesis Magister en Ciencias Agrarias, Universidad Nacional de Colombia, Palmira.

Palencia, G., Mercado, T. y Combatt. E. 2006. Estudio Agro-meteorológico del departamento de Córdoba. Universidad de Córdoba, Montería - Colombia, 126 p.

Prior, S., Runion, G., Marble, C., Rogers, H.,
Gilliam, C. and Torbert, H. 2011. A review of elevated atmospheric $\mathrm{CO} 2$ effects on plant growth and water relations: Implications for Horticulture. Hortscience 46(2): 158-162.

Rebolledo, M., Dingkuhn, M., Péré, P., McNally, K. L. and Luquet, D. 2012. Developmental dynamics and early growth vigour in Rice. I. Relationship between development rate (1/Phyllochron) and growth. Journal of Agronomy and Crop Science 198 (5): 374-384.

Riveros, G. 1994. Fisiología de la producción de arroz. Revista Comalfi 21 (2): 27-34.

Roudier, P., Sultan, B., Quirion, P. and Berg, A. 2011. The impact of future climate change on West African crop yields: What does the recent literature say? Global Environmental Change 21(3): 1073-1083.

SAS. 1999. SAS Software. Version 9.1. Cary, North Carolina: SAS Institute Inc.

Shahidullah, S., Hanafi, M., Ashrafuzzaman, M., Salam, A. and Khair, A. 2009. Flowering response and crop duration of aromatic rices in diverse environments. Comptes Rendus Biologies 332 (10): 909916.

Soto, F. y Hernández, N. 2012. Influencia de tres fechas de siembra en el crecimiento y rendimiento de especies de cereales cultivadas en condiciones tropicales. Parte II. Cultivo del sorgo (Sorghum bicolor L. Moench var. ISIAP Dorado). Cultivos Tropicales 33 (2): 50-55.

Takai, T., Matsuura, S., Nishio, T., Ohsumi, A., Shiraiwa, T. and Horie, T. 2005. Rice yield potential is closely related to crop growth rate during late reproductive period. Field Crops Research 96: 328-335. 